\title{
Electrical control of fine-structure splitting in self-assembled quantum dots for entangled photon pair creation
}

Cite as: Appl. Phys. Lett. 97, 221108 (2010); https://doi.org/10.1063/1.3522655

Submitted: 10 September 2010 . Accepted: 09 November 2010 . Published Online: 01 December 2010

J. D. Mar, X. L. Xu, J. S. Sandhu, A. C. Irvine, M. Hopkinson, and D. A. Williams

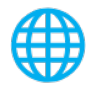

\section{ARTICLES YOU MAY BE INTERESTED IN}

Charge state control in single InAs/GaAs quantum dots by external electric and magnetic fields

Applied Physics Letters 105, 041109 (2014); https://doi.org/10.1063/1.4891828

Strongly coupled single quantum dot in a photonic crystal waveguide cavity

Applied Physics Letters 97, 111101 (2010); https://doi.org/10.1063/1.3487937

Influence of an in-plane electric field on exciton fine structure in InAs-GaAs self-assembled quantum dots

Applied Physics Letters 86, 041907 (2005); https://doi.org/10.1063/1.1855409

\section{Lock-in Amplifiers up to $600 \mathrm{MHz}$

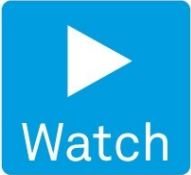

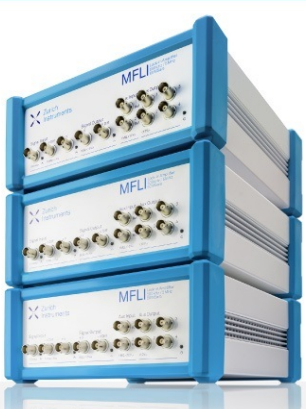




\title{
Electrical control of fine-structure splitting in self-assembled quantum dots for entangled photon pair creation
}

\author{
J. D. Mar, ${ }^{1, a)}$ X. L. Xu, ${ }^{1}$ J. S. Sandhu, ${ }^{1}$ A. C. Irvine ${ }^{2}$ M. Hopkinson, ${ }^{3}$ and D. A. Williams ${ }^{1}$ \\ ${ }^{1}$ Hitachi Cambridge Laboratory, Cavendish Laboratory, Cambridge CB3 OHE, United Kingdom \\ ${ }^{2}$ Microelectronics Research Centre, Cavendish Laboratory, Cambridge CB3 OHE, United Kingdom \\ ${ }^{3}$ Department of Electronic and Electrical Engineering, University of Sheffield, Sheffield S1 3JD, \\ United Kingdom
}

(Received 10 September 2010; accepted 9 November 2010; published online 1 December 2010)

\begin{abstract}
The fine-structure splitting $\delta_{1}$ of the bright exciton doublet in self-assembled InGaAs/GaAs quantum dots is measured as a function of the applied in-plane electric field using time-resolved Kerr rotation. We observe a significant reduction of $\delta_{1}$ with increasing electric field, suggesting a symmetrizing of the electron and hole wave functions. For sufficiently high fields, it is shown that $\delta_{1}$ can be made less than the quantum dot homogeneous linewidth $\gamma$, which is calculated using an appropriate model. This may open the way for the electrical control of polarization-entangled photon pair creation through the radiative biexciton cascade. (C) 2010 American Institute of Physics. [doi:10.1063/1.3522655]
\end{abstract}

A reliable source of entangled photon pairs is a prerequisite for the implementation of quantum information processing (QIP), which includes quantum computation ${ }^{1}$ and quantum cryptography. ${ }^{2}$ Despite its inefficiency, the conventional approach to creating entangled photon pairs has been parametric down-conversion of attenuated laser pulses in a nonlinear optical crystal. Alternatively, due to the long coherence time of its ground-state exciton, it has been proposed that a single self-assembled quantum dot (QD) can be used as an efficient source of polarization-entangled photon pairs through the radiative decay of its biexciton state. ${ }^{3}$

The heavy-hole excitons $(X)$ in a QD have a total angular momentum $M$ composed of the heavy-hole angular momentum $(j= \pm 3 / 2)$ and the electron spin $(s= \pm 1 / 2)$, producing four degenerate exciton states, where states with $M$ $= \pm 1$ (bright excitons) and $M= \pm 2$ (dark excitons) are optically active and inactive, respectively. However, independent of the QD symmetry, the short-range component of the electron-hole exchange interaction results in a splitting of the exciton multiplet into bright and dark exciton doublets. In addition, it mixes the dark states leading to a lifting of their degeneracy. For dots with perfect $D_{2 d}$ symmetry, the bright excitons are degenerate and their radiative recombination leads to $\sigma^{ \pm}$-circularly polarized photons, thus making angular momentum a good quantum number. When the QD ground state is occupied by two excitons having opposite spin orientations, a spin singlet biexciton state $(2 X)$ is created from which two recombination pathways are possible [Fig. 1(a), left side], resulting in the cascaded emission of two photons with opposite circular polarizations. Therefore, this two-photon polarization state can be written as $|\psi|$ $=1 / \sqrt{2}\left(\left|\sigma^{+}\right\rangle_{1}\left|\sigma^{-}\right\rangle_{2}+\left|\sigma^{-}\right\rangle_{1}\left|\sigma^{+}\right\rangle_{2}\right)$, which is an entangled state.

The first experiments attempting to demonstrate such photon-photon entanglement were unsuccessful, showing instead classical correlations. ${ }^{4,5}$ This was due to a mixing of the bright exciton states to create a nondegenerate bright exciton doublet $|+1\rangle \pm|-1\rangle$ referred to as fine-structure splitting

\footnotetext{
a) Author to whom correspondence should be addressed. Electronic mail: jm585@cam.ac.uk.
}

(FSS), as depicted on the right-hand side of Fig. 1(a). Such FSS has been attributed to the fact that realistic selfassembled QDs possess a broken symmetry $\left(<D_{2 d}\right)$ with preferential elongation ${ }^{6}$ and strain ${ }^{7}$ along the $[1-10]$ crystallographic direction, resulting in a modification of the electron-hole overlap and hence a change in the long-range component of the electron-hole exchange interaction, which strongly determines the FSS. ${ }^{8}$ Consequently, the two bright exciton states are distinguishable by the linear polarization of their emission along either the [110] or [1-10] direction ${ }^{9}$ (denoted as $\pi_{y}$ or $\pi_{x}$, respectively), thus making angular momentum not a good quantum number. Since the energy difference in the bright exciton doublet due to the FSS, denoted as $\delta_{1}$, is usually greater than the homogeneous linewidth $\gamma$ of the QD exciton, the two biexciton recombination pathways

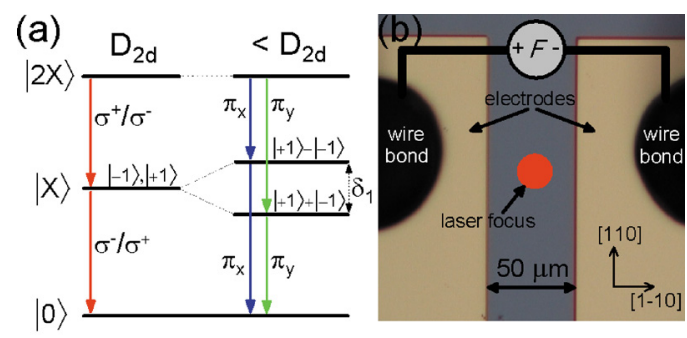

(c)

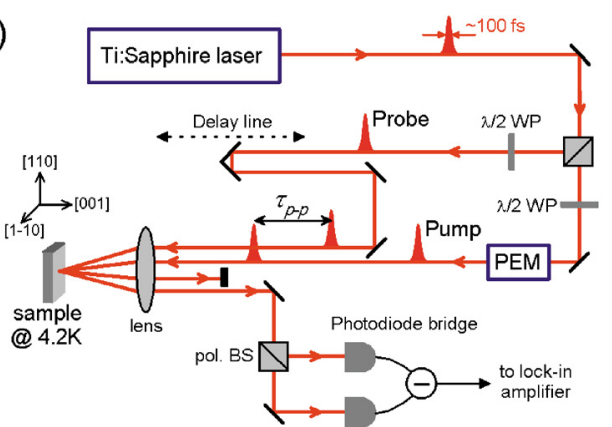

FIG. 1. (Color online) (a) Energy-level scheme showing the possible biexciton $(2 X)$ recombination pathways in a self-assembled QD with $D_{2 d}$ symmetry (left side) and $<D_{2 d}$ symmetry (right side). (b) Optical microscope image of a device. (c) TRKR experimental setup, as described in the text. $\lambda / 2$ WP: half-wave plate; PEM: photoelastic modulator; $\tau_{p-p}$, pump-probe time delay; pol. BS: polarizing beamsplitter. 
can easily be distinguished through a simple energy measurement even without any knowledge of the photon polarizations. In this situation there is clearly no photon-pair entanglement. Thus, it is an essential requirement that $\delta_{1}$ be reduced to less than $\gamma$ in order to make possible the creation of entangled photon pairs through the radiative biexciton cascade.

There have been several approaches undertaken with the goal of reducing $\delta_{1}$ below $\gamma$, notably using thermally annealed QDs (Ref. 10) and applying external magnetic fields. ${ }^{11}$ Although these approaches have resulted in a significant degree of control over the FSS, both are impractical in terms of their ability to tune dynamically the FSS of individual QDs in a scalable QD-based device. Alternatively, it has been demonstrated that applying an in-plane electric field along one of the [110] crystal directions can reduce $\delta_{1}$ (Refs. 12 and 13) by changing the single-particle wave functions and thus modifying the electron-hole overlap, which influences the FSS through the long-range exchange interaction. ${ }^{8}$ Compared to the aforementioned approaches, the ability to tune the FSS using a local electric field would be more practical for the dynamic control of FSS in individual QDs toward a future scalable device. In addition, conventional highdensity gate design technologies could be used to realize high-density arrays of such devices.

To date, studies that have applied an in-plane electric field have observed significant reductions in FSS. ${ }^{12,13}$ However, in these studies $\delta_{1}$ was measured using photoluminescence (PL) spectroscopy, which offers a limited spectral resolution on the scale of interest when attempting to compare $\delta_{1}$ with $\gamma$. In this letter, we use an ultrafast pump-probe technique: time-resolved Kerr rotation (TRKR), ${ }^{14}$ which offers a higher resolution that is limited only by the laser pulse duration $(\sim 100 \mathrm{fs})$ to measure directly the very small values of $\delta_{1}$. Using this technique, we show that the application of an in-plane electric field along the [1-10] direction can indeed reduce $\delta_{1}$ to values comparable to $\gamma$, thus potentially opening the way for creating entangled photon pairs through the radiative biexciton cascade.

Experiments throughout this study were performed on self-assembled InGaAs/GaAs QDs, which were formed in the Stranski-Krastanow growth mode using molecular beam epitaxy. The 7 ML thick QD layers were grown in the middle of a $200 \mathrm{~nm}$ thick GaAs layer, below which is a $100 \mathrm{~nm}$ thick $\mathrm{Al}_{0.3} \mathrm{Ga}_{0.7} \mathrm{As}$ barrier to prevent carrier diffusion. In order to apply an in-plane electric field in the [1-10] direction, two parallel $\mathrm{Au} / \mathrm{Cr}$ electrodes $(450 / 20 \mathrm{~nm}$ thick) were fabricated on the top surface of the sample using electron-beam lithography techniques. As shown schematically in Fig. 1(b), the electrodes are separated by $50 \mu \mathrm{m}$ to ensure sufficient interaction between the incident laser and the QDs during TRKR measurements.

In the TRKR measurements, a mode-locked Ti:sapphire laser acts as a source of $\sim 100$ fs pulses with an energy tuned into resonance with that of the QD exciton transition. Each pulse is divided at a beamsplitter in order to obtain a circularly polarized pump pulse and a linearly polarized probe pulse, which is delayed in time with respect to the pump using a motorized variable delay stage. For a QD with FSS, the $\sigma^{ \pm}$-circularly polarized pump pulse then coherently excites the two nondegenerate linearly polarized eigenstates to create the coherent superposition exciton state: $\Psi(0)$

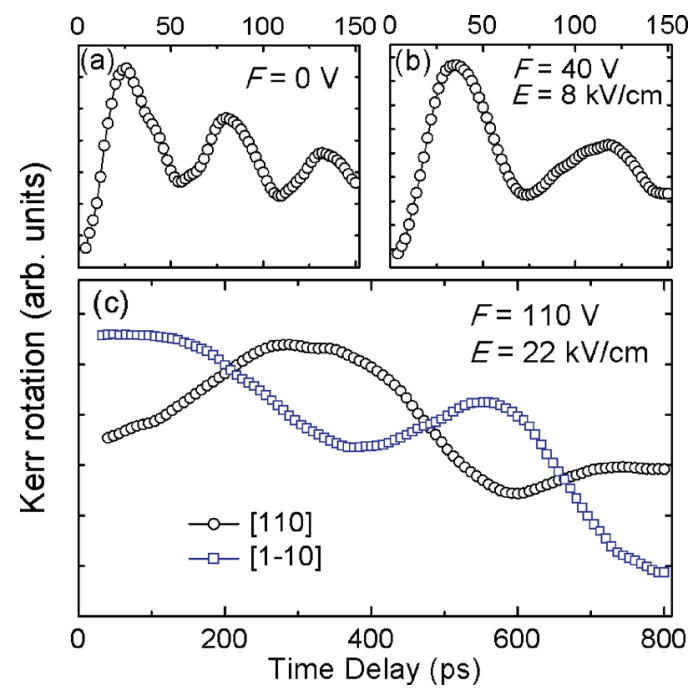

FIG. 2. (Color online) Kerr rotation as a function of pump-probe time delay with incident probe polarization along the [110] crystal direction for (a) $F=0 \mathrm{~V}$ and (b) $F=40 \mathrm{~V}(E=8 \mathrm{kV} / \mathrm{cm})$. (c) TRKR for $F=110 \mathrm{~V}(E$ $=22 \mathrm{kV} / \mathrm{cm}$ ) with the incident probe polarization along the [110] (circles) and [1-10] (squares) crystal directions.

$=\left|\sigma^{ \pm}\right\rangle=1 / \sqrt{2}[(|+1\rangle+|-1\rangle) \pm i(|+1\rangle-|-1\rangle)]$. Due to $\delta_{1}$, this state evolves over time with an average quantum beat frequency $\omega$ according to $e^{i \omega t}|\Psi(t)\rangle=1 / \sqrt{2}\left[e^{-i \omega t / 2}(|+1\rangle+\mid\right.$ $\left.-1\rangle) \pm i e^{+i \omega t / 2}(|+1\rangle-|-1\rangle)\right]=\cos (\omega t / 2)\left|\sigma^{ \pm}\right\rangle-i \sin (\omega t / 2)\left|\sigma^{\mp}\right\rangle$. Since the degree of Kerr rotation of the linearly polarized probe is determined by the exciton polarization at a given pump-probe time delay $\tau_{p-p}$, this time evolution can be measured by varying $\tau_{p-p}$ and is observed as oscillations in the TRKR with the same beat frequency $\omega=\delta_{1} / \hbar$. Therefore, we are able to deduce values for $\delta_{1}$ from the frequency of oscillations in the TRKR. As shown schematically in Fig. 1(c), the sample was held in a He-flow cryostat at a temperature of $4.2 \mathrm{~K}$ and the Kerr rotation of the probe was measured by employing a balanced photodiode bridge and lock-in techniques using a photoelastic modulator (PEM), which modulates the polarization of the pump between orthogonal circular polarizations.

We determined $\delta_{1}$ as a function of in-plane electric field $E$ applied in the [1-10] direction ${ }^{15}$ by measuring the oscillation frequency in the TRKR. Values for $E$ at the QD layers as a function of applied voltage $F$ between the electrodes were calculated using a commercial device simulation package (SILVACO ATLAS). First, Fig. 2(a) shows the zero-field ( $F$ $=0 \mathrm{~V})$ TRKR with the linear polarization of the incident probe along the [110] direction. Clear oscillations are observed here with a period of $56 \pm 2 \mathrm{ps}$, corresponding to $\delta_{1}=74 \pm 2 \mu \mathrm{eV}$. This value of $\delta_{1}$ is comparable to results from past studies using four-wave mixing ${ }^{16}$ and micro-PL ( $\mu$-PL) spectroscopy $^{8}$ on similar QDs. Note that the oscillations are damped as a result of the inhomogeneous distribution of $\delta_{1}$ in the ensemble of QDs and, perhaps to a lesser extent, scattering processes with phonons. ${ }^{17}$ Next, Fig. 2(b) presents the TRKR for an applied bias $F=40 \mathrm{~V}(E$ $=8 \mathrm{kV} / \mathrm{cm}$ ) between the electrodes. Here, we observe an increase in the oscillation period to $80 \pm 2 \mathrm{ps}$, corresponding to a decrease in the FSS to $\delta_{1}=52 \pm 1 \mu \mathrm{eV}$. Finally, the results in Fig. 2(c) were obtained at a high electric field with $F=110 \mathrm{~V}(E=22 \mathrm{kV} / \mathrm{cm})$. For this $F$, we observe a dramatic increase in the oscillation period to $614 \pm 8 \mathrm{ps}$, which 


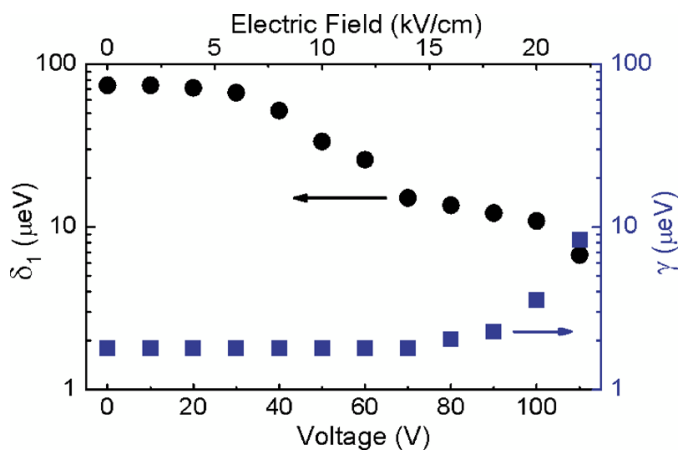

FIG. 3. (Color online) Dependence of $\delta_{1}$ (circles) and homogeneous linewidth $\gamma$ (squares) on applied voltage $F$ and its corresponding in-plane electric field $E$.

corresponds to a significant reduction in the FSS to $\delta_{1}$ $=6.7 \pm 0.1 \mu \mathrm{eV}$. Also, when the incident probe linear polarization is rotated by $90^{\circ}$ (i.e., rotated to the [1-10] direction), the oscillations undergo a $\pi$-phase shift, thereby confirming the authenticity of the observed oscillations. Figure 3 summarizes our results for $\delta_{1}$ (circles) as a function of applied voltage $F$ and its corresponding electric field $E$.

The homogeneous linewidth of the ground state exciton transition in self-assembled QDs and its broadening due to the presence of high electric fields have been modeled extensively and supported experimentally throughout the literature. ${ }^{18,19}$ Increasing the applied electric field reduces the tunneling barrier width, resulting in a dynamic contest between carrier tunneling out of the QD and exciton recombination. The increase in carrier tunneling leads to a decrease in the exciton lifetime in the QD and, in turn, results in a broadening of the QD homogeneous linewidth $\Gamma$. This phenomenon can be accurately modeled using a onedimensional (1-D) Wentzel-Kramers-Brillouin (WKB) approximation, ${ }^{18,19}$ considering the carrier as a particle in a rectangular confining potential of lateral extension $a$ and height $V_{0}$ (Ref. 20)

$$
\Gamma \geq \frac{\pi \hbar^{2}}{2 m^{*} a^{2}} \exp \left(-\frac{4 \sqrt{2 m^{*}}}{3 \hbar} \frac{V_{0}^{3 / 2}}{e F}\right)+\Gamma_{0},
$$

where $\Gamma_{0}$ is the linewidth for zero electric field and $m^{*}$ is the electron effective mass in the GaAs tunneling barrier. Values for $a$ and $\Gamma_{0}$ were taken from past work on QDs from the same material composition and growth mode. ${ }^{21,22}$ Previous studies investigating the effect of an electric field on homogeneous linewidth have shown that the above model is readily consistent with experimental data. ${ }^{18,19}$

Using this model, Fig. 3 summarizes the results for $\gamma$ alongside those for $\delta_{1}$ as a function of applied voltage $F$ and its corresponding in-plane electric field $E$. For low voltages $(F \leq 30 \mathrm{~V})$, we see that $\delta_{1}$ remains considerably large and is greater than $\gamma$ by a factor of about 40. However, when the voltage is increased beyond this range, a rapid decrease in $\delta_{1}$ is observed due to the applied in-plane electric field, whereas $\gamma$ is still relatively unchanged for such intermediate voltages. At high voltages $(F>80 \mathrm{~V}), \delta_{1}$ is significantly reduced while $\gamma$ is increased to values on the same order of magnitude as $\delta_{1}$. Most importantly, at an applied voltage of $F$ $=110 \mathrm{~V}(E=22 \mathrm{kV} / \mathrm{cm})$, we observe a situation where $\delta_{1}$ is decreased to $6.7 \pm 0.1 \mu \mathrm{eV}$ while $\gamma$ is increased to $8.3 \mu \mathrm{eV}$, according to the model. Here, we see that $\delta_{1}$ has been reduced to a value less than $\gamma$, suggesting that it may be pos- sible to tune electrically the FSS for the realization of polarization-entangled photon pairs through the radiative biexciton cascade. Moreover, $\gamma$ may be further increased by reducing the exciton lifetime using larger QDs and/or, together with the Purcell effect, QDs placed in an optical resonator which is resonant with the exciton transition energy.

In conclusion, we have shown that it is indeed possible to reduce the FSS of the bright exciton doublet in selfassembled QDs through the application of an in-plane electric field. By using TRKR to obtain precise values of $\delta_{1}$ and using an appropriate model to calculate $\gamma$, we found that for sufficiently high electric fields it may be feasible to reduce $\delta_{1}$ to values below $\gamma$, which would open the way for achieving electrically controlled entangled photon pair creation for applications in QIP.

The authors wish to acknowledge R. T. Phillips and W. J. H. Leyland for valuable and helpful discussions.

${ }^{1}$ E. Knill, R. Laflamme, and G. J. Milburn, Nature (London) 409, 46 (2001).

${ }^{2}$ N. Gisin, G. Ribordy, W. Tittel, and H. Zbinden, Rev. Mod. Phys. 74, 145 (2002).

${ }^{3}$ O. Benson, C. Santori, M. Pelton, and Y. Yamamoto, Phys. Rev. Lett. 84, $2513(2000)$

${ }^{4}$ C. Santori, D. Fattal, M. Pelton, G. Solomon, and Y. Yamamoto, Phys. Rev. B 66, 045308 (2002).

${ }^{5}$ R. M. Stevenson, R. M. Thompson, A. J. Shields, I. Farrer, B. E. Kardynal, D. A. Ritchie, and M. Pepper, Phys. Rev. B 66, 081302 (2002).

${ }^{6}$ D. Bimberg, M. Grundmann, and N. N. Ledenstov, Quantum Dot Heterostructures (Wiley, Chichester, 1999).

${ }^{7}$ G. Bester, S. Nair, and A. Zunger, Phys. Rev. B 67, 161306(R) (2003).

${ }^{8}$ M. Bayer, G. Ortner, O. Stern, A. Kuther, A. A. Gorbunov, A. Forchel, P. Hawrylak, S. Fafard, K. Hinzer, T. L. Reinecke, S. N. Walck, J. P. Reithmaier, F. Klopf, and F. Schafer, Phys. Rev. B 65, 195315 (2002).

${ }^{9}$ M. Bayer, A. Kuther, A. Forchel, A. Gorbunov, V. B. Timofeev, F. Schäfer, J. P. Reithmaier, T. L. Reinecke, and S. N. Walck, Phys. Rev. Lett. 82, 1748 (1999).

${ }^{10}$ A. Greilich, M. Schwab, T. Berstermann, T. Auer, R. Oulton, D. R. Yakovlev, M. Bayer, V. Stavarache, D. Reuter, and A. Wieck, Phys. Rev. B 73, 045323 (2006).

${ }^{11}$ R. M. Stevenson, R. J. Young, P. See, D. G. Gevaux, K. Cooper, P. Atkinson, I. Farrer, D. A. Ritchie, and A. J. Shields, Phys. Rev. B 73, 033306 (2006).

${ }^{12}$ K. Kowalik, O. Krebs, A. Lemaitre, S. Laurent, P. Senellart, P. Voisin, and J. A. Gaj, Appl. Phys. Lett. 86, 041907 (2005).

${ }^{13}$ S. M. Ulrich, R. Hafenbrak, M. M. Vogel, L. Wang, A. Rastelli, O. G. Schmidt, and P. Michler, Phys. Status Solidi B 246, 302 (2009).

${ }^{14}$ J. J. Baumberg, D. D. Awschalom, and N. Samarth, J. Appl. Phys. 75, 6199 (1994)

${ }^{15}$ There was no observed in-plane electric field dependence of $\delta_{1}$ for identical measurements performed with fields applied along the [110] crystal direction.

${ }^{16}$ W. Langbein, P. Borri, U. Woggon, V. Stavarache, D. Reuter, and A. D. Wieck, Phys. Rev. B 69, 161301 (2004).

${ }^{17}$ A. I. Tartakovskii, M. N. Makhonin, I. R. Sellers, J. Cahill, A. D. Andreev, D. M. Whittaker, J.-P. R. Wells, A. M. Fox, D. J. Mowbray, M. S Skolnick, K. M. Groom, M. J. Steer, H. Y. Liu, and M. Hopkinson, Phys. Rev. B 70, 193303 (2004).

${ }^{18}$ W. Heller, U. Bockelmann, and G. Abstreiter, Phys. Rev. B 57, 6270 (1998)

${ }^{19}$ R. Oulton, J. J. Finley, A. D. Ashmore, I. S. Gregory, D. J. Mowbray, M. S. Skolnick, M. J. Steer, S.-L. Liew, M. A. Migliorato, and A. J. Cullis, Phys. Rev. B 66, 045313 (2002).

${ }^{20} V_{0}=54 \mathrm{meV}$ is defined as the energy offset between the center of the QD ensemble ground state emission and the lateral barrier (wetting layer), and was determined through PL measurements.

${ }^{21}$ H. Drexler, D. Leonard, W. Hansen, J. P. Kotthaus, and P. M. Petroff, Phys. Rev. Lett. 73, 2252 (1994).

${ }^{22}$ M. Bayer and A. Forchel, Phys. Rev. B 65, 041308 (2002). 